\title{
OR-72
}

\section{Brahmi, a Medicinal Plant for Memory Improvement}

\author{
Kornkanok Ingkaninan ${ }^{1, *}$, Nanteetip Limpeanchop ${ }^{1}$, Sakchai Wittaya-areekul ${ }^{1}$, Krongkarn Chootip ${ }^{2}$, \\ Pornnarin Taepavarapruk ${ }^{2}$, Nuwat Taepavarapruk ${ }^{2}$, Jintanaporn Wattanathorn ${ }^{3}$, Seewaboon \\ Sireeratawong ${ }^{4}$, Waraporn Putalun ${ }^{5}$, Watoo Phrompittayarat ${ }^{6}$ and Hiroyuki Tanaka ${ }^{7}$
}

\begin{abstract}
${ }^{1}$ Faculty of Pharmaceutical Sciences and Center of Excellence for Innovation in Chemistry, Naresuan University, Phitsanulok 65000, Thailand; '2Department of Physiology, Faculty of Medical Sciences, Naresuan University, Phitsanulok 65000, Thailand; ${ }^{3}$ Department of Physiology, Faculty of Medicine, Khon Kaen University, Khon Kaen 40002, Thailand; ${ }^{4}$ Department of Preclinical Science, Faculty of Medicine, Thammasat University, Pathumthani. 12120, Thailand; ${ }^{5}$ Faculty of Pharmaceutical Sciences, Khon Kaen University, Khon Kaen 40002, Thailand; ${ }^{6}$ Department of Pharmacognosy and Pharmaceutical Chemistry, Faculty of Pharmacy, Srinakharinwiroj University, 26120 Nakhonayok, Thailand; 7 Department of Medicinal Plant Breeding, Graduate School of Pharmaceutical Sciences, Kyushu University.Fukuoka 812-8582,Japan; E-mail: k_ingkaninan@yahoo.com
\end{abstract}

Brahmi (Bacopa monnieri Wettst., Schrophulariaceae) has been used in Ayuravedic medicine as memory enhancer. We aimed at research and development of Brahmi for clinical use as the food supplement for memory improvement. The preparation and quality control methods for Brahmi extract have been developed. The in vivo studies indicated that the standardized extract had neuroprotective effect at the doses of 40 and $80 \mathrm{mg} / \mathrm{kg} \mathrm{BW}$. The escape latency time of rats in Morris water maze test was reduced. Moreover, the reduction of neurons and cholinergic neuron densities were mitigated. We also tested its protection effect against the beta-amyloid protein and glutamate-induced neurotoxicity in primary cortical cultured neurons. Our result demonstrated that Brahmi extract protected neurons from beta-amyloid-induced cell death, but not glutamate-induced excitotoxicity. The study the effect of Brahmi extract on learning and memory in the middle aged and elderly volunteer was conducted. The results showed that Brahmi extract (300 and $600 \mathrm{mg} /$ day) could improve quality of life in the elderly by improving the physical fitness via the increase in efficiency of postural balance. It also improved the psychological fitness by increasing alertness and attention resulted in the improvement of learning and memory. In addition, Brahmi extract could decrease depression-like symptom. The dosage used in this study did not produce toxic and side effects. Therefore, Brahmi extract may have the potential to be used as food supplement and used as the adjuvant therapy for various conditions including learning and memory impairment, attention deficit syndrome, depression and postural balance impairment.

Keywords: Bacopa monnieri, memory, neuroprotective, saponin glycosides. 